CLINICAL STUDY

\title{
Impaired glucose metabolism in Japanese patients with acromegaly is restored after successful pituitary surgery if pancreatic $\beta$-cell function is preserved
}

\author{
Yuka Kinoshita $^{1,3}$, Hiroko Fujii ${ }^{1,4}$, Akira Takeshita ${ }^{1,5}$, Manabu Taguchi $^{1,3}$, Megumi Miyakawa ${ }^{1,5}$, Kenich Oyama $^{2}$, \\ Shozo Yamada ${ }^{2,5}$ and Yasuhiro Takeuchi ${ }^{1,5}$ \\ ${ }^{1}$ Division of Endocrinology and ${ }^{2}$ Pituitary Surgery, Toranomon Hospital Endocrine Center, 2-2-2 Toranomon Minato-ku, Tokyo 105-8470, Japan, \\ ${ }^{3}$ Department of Nephrology and Endocrinology, University of Tokyo School of Medicine, Tokyo 113-8655, Japan, ${ }^{4}$ Division of Endocrinology and \\ Metabolism, Department of Internal Medicine, National Defense Medical College, Tokorozawa 359-8513, Japan and ${ }^{5}$ Okinaka Memorial Institute for \\ Medical Research, Tokyo 105-8470, Japan
}

(Correspondence should be addressed to Y Takeuchi at Division of Endocrinology, Toranomon Hospital Endocrine Center; Email: takeuchi-tky@umin.ac.jp)

\begin{abstract}
Objective: Impaired glucose metabolism is common in acromegaly, but it is not clear how glucose metabolism is impaired or what predicts its restoration after cure of the disease. To identify factors involved in the impairment of glucose metabolism in acromegaly, we evaluated clinical parameters before and immediately after surgical cure of the disease.

Design and methods: We retrospectively analyzed clinical data of 92 consecutive Japanese patients with acromegaly who underwent successful pituitary surgery. Patients who had received medical therapy for acromegaly or insulin treatment for diabetes were excluded. We evaluated insulin resistance (IR) and pancreatic $\beta$-cell function in addition to GH and IGF1 levels before and after surgery.

Results: In this study of Japanese patients with acromegaly, average body mass index (BMI) was 23.4, and no patient had a BMI $>30$. IR was involved in the impairment of glucose metabolism, which was restored upon surgical cure of acromegaly if $\beta$-cell function was preserved. Insufficient $\beta$-cell function did not improve after normalization of GH/IGF1 and was associated with impaired glucose metabolism before and after surgery. Results of receiver operating characteristic analysis of preoperative clinical parameters suggest that insulinogenic index (IGI) $>0.50$ best predicts restoration of normal glucose metabolism upon cure of acromegaly in Japanese patients.

Conclusions: IR impairs glucose metabolism in acromegaly. Once $\beta$-cell function is impaired, abnormal glucose metabolism persists even after cure of acromegaly. IGI $>0.50$ indicates that $\beta$-cell function is preserved in non-obese Japanese patients with acromegaly.
\end{abstract}

European Journal of Endocrinology 164 467-473

\section{Introduction}

Acromegaly is generally caused by a benign GH-secreting pituitary adenoma. GH stimulates target tissues to synthesize and secrete insulin-like growth factor 1 (IGF1) (1). Elevated serum GH and IGF1 levels are correlated with mortality in acromegaly (2), and excess $\mathrm{GH}$ is believed to cause impaired glucose metabolism, including diabetes mellitus (DM), as well as skeletal complications and cardiovascular and respiratory diseases (3). Impaired glucose metabolism is associated with excess mortality in acromegaly by promoting atherosclerosis, which results in cardiovascular and cerebrovascular diseases.

The prevalence of DM among patients with acromegaly has been reported to range from 19 to $56 \%$ (3). Risk factors for impaired glucose metabolism include elevated serum GH levels, older age, longer duration of disease, family history of DM, and concomitant hypertension (4-6). Kasayama et al. (7) reported defects in pancreatic $\beta$-cell function associated with impaired glucose tolerance (IGT) in patients with acromegaly. In their study, patients with acromegaly generally exhibited insulin resistance (IR) with or without IGT, whereas sufficient insulin secretion was observed in patients with normal glucose tolerance (NGT) to overcome their IR (7). Studies have investigated impaired glucose metabolism in acromegaly, but it is unclear which clinical parameters (e.g. GH, IGF1) play a role in IR and/or impaired $\beta$-cell function in the disease $(7-10)$.

In particular, little is known about the effect of successful surgical treatment for acromegaly on glucose metabolism in patients with IGT or DM. Surgery does not restore normal glucose metabolism in all patients, 
although IR is generally improved (6). So far, it is unclear which clinical parameters determine the restoration of normal glucose metabolism, and whether this outcome can be predicted before surgery.

We therefore analyzed clinical data including age, hormone levels, and indicators of IR and pancreatic $\beta$-cell function in patients with acromegaly before and within a month after surgery to determine their association with glucose metabolism.

\section{Subjects and methods}

\section{Subjects}

Ninety-two consecutive Japanese patients with acromegaly (46 men and 46 women) were enrolled in the present study. All patients were examined and treated at Toranomon Hospital (Tokyo, Japan) from 2005 to 2008. Data regarding routine medical procedures were collected retrospectively. The present study was approved by the institutional review board of Toranomon Hospital.

The diagnosis of acromegaly was based on clinical findings, failure of serum GH suppression during a 75-g oral glucose tolerance test (OGTT), and elevated levels of IGF1 after adjusting for age and sex. Pituitary adenoma was confirmed by magnetic resonance imaging, and transsphenoidal pituitary adenomectomy was performed by expert neurosurgeons. Within 1 month after surgery, patients underwent the 75-g OGTT. Only patients who met the criteria for cure of acromegaly according to the Cortina consensus statement (11) were enrolled in the present study. Patients were excluded from the study if they had received injections of any somatostatin analog and/or oral dopamine agonist for acromegaly or insulin therapy for DM.

During the study period, 421 patients underwent surgery for the first time, and the number of cured cases immediately after surgery was 268 . Among those who met the criteria for cure of the disease, 113 patients were excluded because not all data were available for analysis. Furthermore, 63 patients were excluded because of medication for acromegaly or insulin therapy before surgery. Among them, only three patients were treated with insulin injection. The demographic data of these 63 patients (age, 47.1 \pm 14.2 years; body mass index (BMI), 23.2 \pm 3.8 ) and levels of HbAlc (6.0 \pm 0.9 ), a marker for glucose metabolism, were similar to that of the analyzed cases (Table 1).

In all patients, glucose tolerance status was evaluated by a 75-g OGTT. Using the WHO criteria, NGT was diagnosed when blood glucose levels were $<110 \mathrm{mg} / \mathrm{dl}$ before glucose loading and $<140 \mathrm{mg} / \mathrm{dl} 2 \mathrm{~h}$ after glucose loading. DM was diagnosed when fasting blood glucose was $>126 \mathrm{mg} / \mathrm{dl}$ or blood glucose was $>200 \mathrm{mg} / \mathrm{dl} 2 \mathrm{~h}$ after glucose loading. The remaining patients received the diagnosis of IGT. Patients were
Table 1 Baseline characteristics stratified by preoperative glucose tolerance status. Results are expressed as mean \pm s.D.

\begin{tabular}{lccc}
\hline & NGT & IGT & DM \\
\hline Patients $(n)$ & 39 & 36 & 17 \\
Sex (M/F) & $20 / 19$ & $18 / 18$ & $8 / 9$ \\
Age (years) & $43.9 \pm 12.4$ & $46.2 \pm 14.1$ & $51.1 \pm 11.3$ \\
Body mass index & $23.6 \pm 2.9$ & $23.2 \pm 2.6$ & $23.1 \pm 3.1$ \\
Fasting GH (ng/ml) & $15.1 \pm 17.7$ & $12.4 \pm 9.4$ & $12.1 \pm 8.4$ \\
IGF1 (ng/ml) & $672.6 \pm 251.4$ & $630.8 \pm$ & $656.4 \pm 213.7$ \\
& & 260.7 & \\
IGF1 (Z-score) & $7.2 \pm 2.5$ & $6.7 \pm 2.5$ & $7.3 \pm 2.2$ \\
HOMA-\% 3 & $102.1 \pm 28.0$ & $97.6 \pm 38.1$ & $68.9 \pm 29.1^{*, \dagger}$ \\
IGI & $1.3 \pm 0.8$ & $0.9 \pm 0.6^{\ddagger}$ & $0.3 \pm 0.3^{*, \dagger}$ \\
HOMA-\%S & $96.2 \pm 49.1$ & $97.0 \pm 74.9$ & $101.5 \pm 48.3$ \\
HOMA-IR & $1.3 \pm 0.6$ & $1.7 \pm 1.2$ & $1.4 \pm 1.1$ \\
HbA1c (\%) & $5.6 \pm 0.4$ & $5.7 \pm 0.4$ & $6.5 \pm 1.2^{*, \dagger}$ \\
\hline
\end{tabular}

$\mathrm{DM}$, diabetes mellitus; HOMA-\% $\beta$, homeostatic model assessment of pancreatic $\beta$-cell function; HOMA-\%S, indicator of insulin sensitivity; HOMA-IR, indicator of insulin resistance; IGF1, insulin-like growth factor 1; IGI, insulinogenic index; IGT, impaired glucose tolerance; NGT, normal glucose tolerance. To convert values for insulin to $\mathrm{pM} / \mathrm{l}$, multiply by 6.945 . To convert values of IGI to $\mathrm{pM} / \mathrm{mM}$, multiply by 125 . ${ }^{*} P<0.01$ compared with NGT. ${ }^{\dagger} P<0.01$ compared with IGT. ${ }^{\ddagger} P<0.05$ compared with NGT.

divided into three groups according to the preoperative and postoperative glucose tolerance status: group I, patients with NGT before surgery (NGT before surgery; $n=39$ ); group II, patients with IGT or DM before surgery, but in whom NGT was restored after surgery (restored NGT after surgery; $n=27$ ); group III, patients with persistent IGT or DM before and after surgery (persistent IGT/DM; $n=26$; Fig. 1). Three patients with DM before and after surgery were treated with oral hypoglycemic agents (sulfonylurea, nateglinide, or metformin).

\section{Data analysis}

All patients underwent a 75-g OGTT after an overnight fast. Blood was collected at baseline and

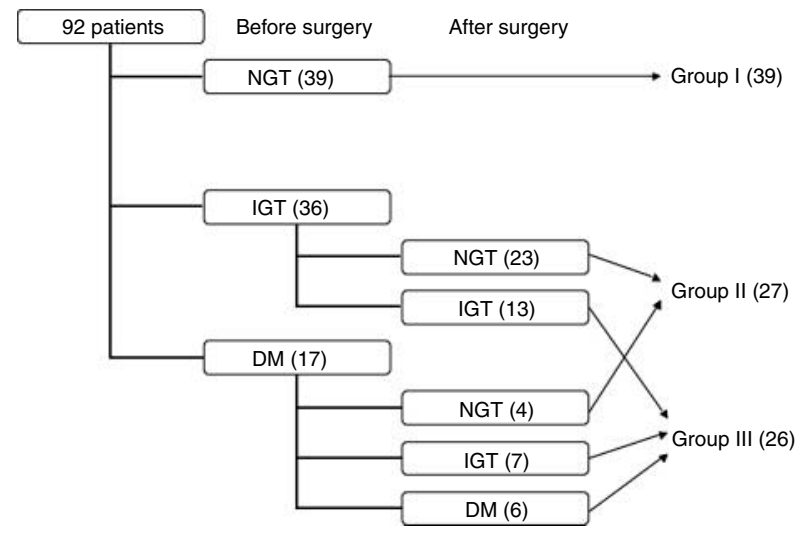

Figure 1 Categorization of patients into three groups according to preoperative and postoperative glucose tolerance status. DM, diabetes mellitus; IGT, impaired glucose tolerance; NGT, normal glucose tolerance. 
then at 30, 60, 90, and 120 min after glucose ingestion, which was assayed for insulin, glucose, and GH. We used homeostatic model assessment (HOMA) computer software to estimate pancreatic $\beta$-cell function (HOMA-\% $\beta$ ), insulin sensitivity (HOMA-\%S), and IR (HOMA-IR) from fasting blood glucose and serum insulin levels $(12,13)$. Because there is a reciprocal relationship between HOMA-\%S and HOMA-IR, we refer to HOMA-\%S as an indicator of IR. Insulinogenic index (IGI) is an OGTT-based estimate of early-phase insulin secretion, calculated as (insulin at $30 \mathrm{~min}-$ insulin at $0 \mathrm{~min}(\mu \mathrm{U} / \mathrm{ml})) /($ glucose at $30 \mathrm{~min}-$ glucose at $0 \mathrm{~min}$ $(\mathrm{mg} / \mathrm{dl}))(14)$. IGF1 values were transformed into age-adjusted and sex-adjusted Z-scores.

\section{Laboratory tests}

Insulin was determined by a chemiluminescent enzyme immunoassay (LUMIPULSE Presto insulin, Fujirebio, Inc., Tokyo, Japan). Blood glucose was determined by the hexokinase method (Liquitech Glucose HK Test, Roche Diagnostics K K). GH was measured with a fluorescent enzyme immunoassay (ST AIA-PACK HGH, Tosoh Co., Tokyo, Japan). IGF1 was measured with an IRMA (IGF1 IRMA Daiichi, TFB Co., Tokyo, Japan).

\section{Statistical analysis}

Results are expressed as mean \pm s.D. Differences among patient groups were evaluated by Fisher's protected least significant difference test. Clinical parameters were evaluated by Pearson's correlation coefficient analysis. Receiver operating characteristic (ROC) analysis was used to evaluate the ability of parameters to predict postoperative glucose tolerance status. The various parameters were compared by calculating the area under the curve (AUC), according to the method of Hanley \& McNeil $(15,16)$. All analyses were carried out with STATVIEW 5.0 software (Abacus Concepts, Inc., Berkeley, CA, USA). The two-tailed $P$ value $<0.05$ was considered significant.

\section{Results}

\section{Clinical parameters and their association with glucose tolerance before surgery}

Table 1 shows the baseline characteristics of patients stratified by preoperative glucose tolerance status: NGT $(n=39)$, IGT $(n=36)$, or DM $(n=17)$. Age, BMI, serum levels of fasting GH and IGF1, and IGF1 Z-scores did not differ significantly among groups. Similarly, the parameters of IR (HOMA-\%S and HOMA-IR) did not differ significantly among groups. However, pancreatic $\beta$-cell function and capacity to secrete insulin (HOMA- $\% \beta$ and IGI) were both significantly lower in patients with DM than those with NGT or IGT (Table 1). IGI was lower in the IGT group than in the NGT group, and HbA1c was higher in the DM group than in the NGT and IGT groups, but did not differ between the NGT and IGT groups.

We then analyzed the relationships among clinical parameters (Table 2). Parameters of $\beta$-cell function (HOMA- $\% \beta$ and IGI) correlated with each other, and both HOMA- $\% \beta$ and IGI positively correlated with IR (HOMA-IR) and negatively correlated with HOMA-\%S. The positive correlation between $\beta$-cell function and IR suggested that IR is the determinant of $\beta$-cell function in acromegaly. Serum levels of GH correlated positively with the IGF1 Z-score and negatively with age; conversely, HOMA-\%S correlated negatively with the IGF1 Z-score and positively with age; HOMA-IR did not correlate with either parameter. BMI correlated positively with HOMA-\% $\beta$, HOMA-IR, and IGF1 Z-score, but was not significantly associated with IGI, HOMA-\%S, or GH (Table 2).

\section{Association of preoperative clinical parameters with glucose tolerance after surgery}

Normal glucose metabolism was restored in some patients but not others after successful surgery for acromegaly; therefore, patients were divided into three groups according to their glucose tolerance before and

Table 2 Pearson's correlation matrix clinical parameters before surgery.

\begin{tabular}{|c|c|c|c|c|c|c|c|c|}
\hline & НОМА-\% $\beta$ & IGI & HOMA-\%S & HOMA-IR & GH & $\begin{array}{c}\text { IGF1 } \\
\text { (Z-score) }\end{array}$ & Age & BMI \\
\hline $\begin{array}{l}\text { HOMA-\% } \\
\text { IGI } \\
\text { HOMA-\%S } \\
\text { HOMA-IR } \\
\text { GH } \\
\text { IGF1 (Z-score) } \\
\text { Age } \\
\text { BMI }\end{array}$ & 1 & $\begin{array}{l}0.538^{*} \\
1\end{array}$ & $\begin{array}{l}-0.700^{*} \\
-0.340^{*} \\
1\end{array}$ & $\begin{array}{r}0.735^{\star} \\
0.289^{*} \\
-0.735^{\star} \\
1\end{array}$ & $\begin{array}{r}0.201 \\
0.147 \\
-0.205 \\
0.103 \\
1\end{array}$ & $\begin{array}{l}0.149 \\
0.151 \\
-0.237^{*} \\
0.151 \\
0.340^{*} \\
1\end{array}$ & $\begin{array}{l}-0.383^{\star} \\
-0.154 \\
0.269^{\star} \\
-0.182 \\
-0.226^{\star} \\
-0.115 \\
1\end{array}$ & $\begin{array}{r}0.264^{\star} \\
0.184 \\
-0.289 \\
0.356^{\star} \\
-0.083 \\
0.260^{\star} \\
-0.069 \\
1\end{array}$ \\
\hline
\end{tabular}

BMI, body mass index; HOMA-\% $\beta$, homeostatic model assessment of pancreatic $\beta$-cell function; HOMA-\%S, indicator of insulin sensitivity; HOMA-IR, indicator of insulin resistance; IGF1, insulin-like growth factor 1 ; IGI, insulinogenic index. ${ }^{\star} P<0.05$. To convert values for insulin to pM/l, multiply by 6.945 . To convert values of IGI to $\mathrm{pM} / \mathrm{mM}$, multiply by 125 . 
after surgery: group I (NGT before surgery), group II (restored NGT after surgery), and group III (persistent IGT/DM; Table 3). We assessed clinical parameters in all patients before surgery. Age, BMI, fasting serum GH, IGF1, and Z-score of IGF1 did not differ significantly among patient groups. However, $\beta$-cell function, HOMA- $\% \beta$, and IGI were significantly lower in group III than in groups I and II (Table 3). Regarding IR, HOMA-IR was significantly higher in group II than in groups I and III, whereas HOMA-\%S did not differ significantly among groups (Table 3), and HOMA-IR was similar in groups I and III.

\section{Analysis of clinical parameters after surgery}

To determine how clinical parameters associated with glucose metabolism changed after surgical treatment of acromegaly, we compared the postoperative parameters of IR and $\beta$-cell function among patient groups (Fig. 2A-C). Compared with preoperative values, HOMA- $\% S$ was increased and HOMA-IR was decreased in all groups $(P<0.05)$, indicating improved IR in all patient groups after surgery. Among parameters of $\beta$-cell function, HOMA- $\% \beta$ was decreased significantly in groups I and II, but IGI was reduced significantly only in group II $(P<0.05$; Fig. 2A and B). Thus, improved IR appeared to be associated with decreased parameters of $\beta$-cell function, at least among patients in groups I and II, supporting the idea that IR is the determinant of $\beta$-cell function in acromegaly.

Postoperative values of HOMA-\%S and HOMA-IR were similar across patient groups (Fig. 2A-C); however, the parameters of $\beta$-cell function (HOMA- $\% \beta$ and IGI) were significantly lower after surgery in group III than in group I (Fig. 2A and C).

Table 3 Preoperative clinical parameters in patients according to glucose tolerance before and after successful surgery for acromegaly. Results are expressed as mean \pm s.D.

\begin{tabular}{lccc}
\hline & $\begin{array}{c}\text { NGT before } \\
\text { surgery } \\
\text { (group I) }\end{array}$ & $\begin{array}{c}\text { Restored } \\
\text { NGT } \\
\text { after surgery } \\
\text { (group II) }\end{array}$ & $\begin{array}{c}\text { Persistent } \\
\text { IGT/DM } \\
\text { (group III) }\end{array}$ \\
\hline Patients $(n)(92)$ & 39 & 27 & 26 \\
Sex (M/F) (46/46) & $20 / 19$ & $15 / 12$ & $11 / 15$ \\
Age (years) & $43.9 \pm 12.4$ & $45.7 \pm 13.7$ & $50.0 \pm 12.9$ \\
Body mass index & $23.6 \pm 2.9$ & $23.5 \pm 2.4$ & $22.7 \pm 3.0$ \\
Fasting GH (ng/ml) & $15.1 \pm 17.7$ & $11.5 \pm 8.2$ & $13.2 \pm 9.9$ \\
IGF1 (ng/ml) & $672.6 \pm 251.4$ & $615.4 \pm 225.1$ & $663.4 \pm 266.1$ \\
IGF1 (Z-score) & $7.2 \pm 2.5$ & $6.5 \pm 2.2$ & $7.3 \pm 2.7$ \\
HOMA-\% $\beta$ & $102.1 \pm 28.0$ & $102.9 \pm 34.5$ & $73.4 \pm 35.5^{*, \dagger}$ \\
IGI & $1.3 \pm 0.8$ & $1.0 \pm 0.6$ & $0.4 \pm 0.4^{*, \dagger}$ \\
HOMA-\%S & $96.2 \pm 49.1$ & $83.5 \pm 61.8$ & $114.0 \pm 69.9$ \\
HOMA-IR & $1.3 \pm 0.6$ & $1.9 \pm 1.3^{*}$ & $1.3 \pm 1.1^{\dagger}$ \\
\hline
\end{tabular}

HOMA-\% $\beta$, homeostatic model assessment of pancreatic $\beta$-cell function; HOMA-\%S, indicator of insulin sensitivity; HOMA-IR, indicator of insulin resistance; IGF1, insulin-like growth factor 1; IGI, insulinogenic index, NGT, normal glucose tolerance. To convert values for insulin to $\mathrm{pM} / \mathrm{l}$, multiply by 6.945. To convert values of IGI to $\mathrm{pM} / \mathrm{mM}$, multiply by 125 . ${ }^{\star} P<0.05$ compared with group I. ${ }^{\dagger} P<0.05$ compared with group II.
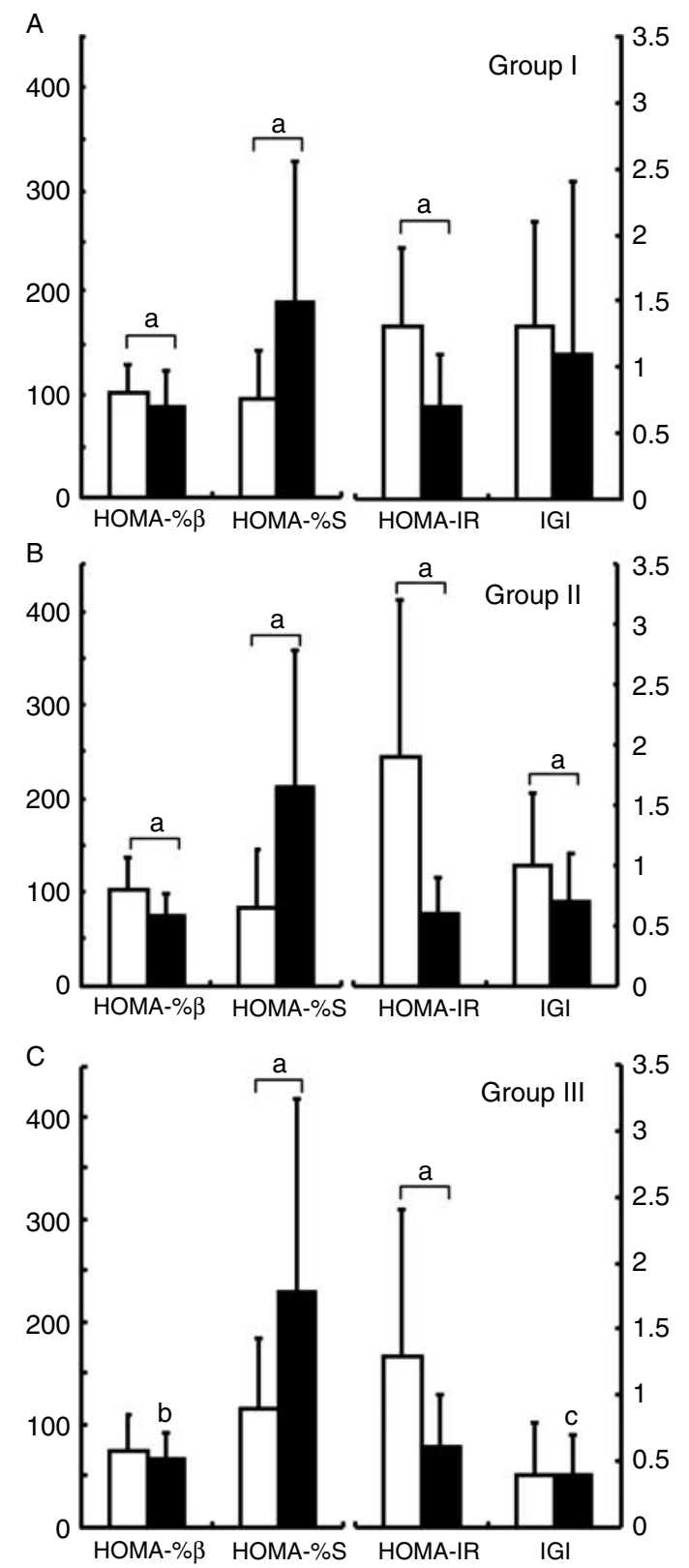

Figure 2 Clinical parameters of patients before surgery for acromegaly (open column) and after surgery (closed column) according to glucose tolerance status. ${ }^{\mathrm{a}} P<0.05$ compared with preoperative values. ${ }^{b} P<0.05$ compared with group I. ${ }^{\mathrm{C}} P<0.01$ compared with group I.

\section{Association of preoperative clinical parameters with improved glucose tolerance after surgery}

Some preoperative parameters (HOMA- $\% \beta$, IGI, and HOMA-IR) were significantly lower in group III than in group II (Table 3). Logistic regression analysis including age, BMI, GH, IGF1, and parameters of IR and $\beta$-cell function was carried out to identify parameters 


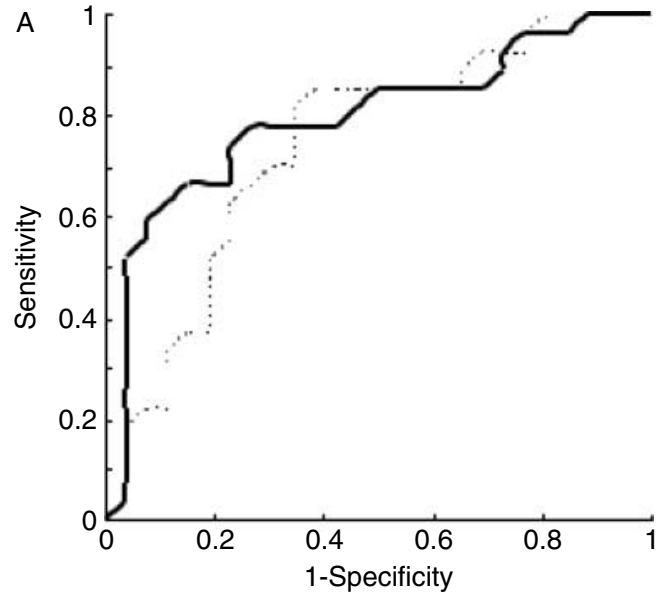

B

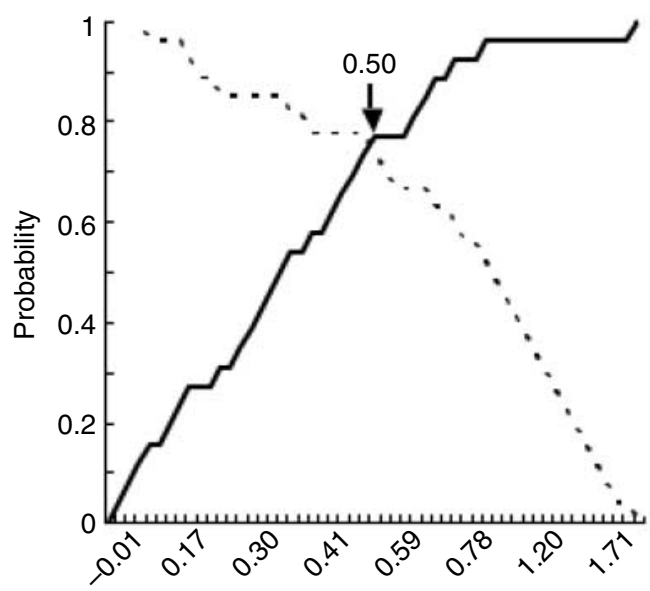

C

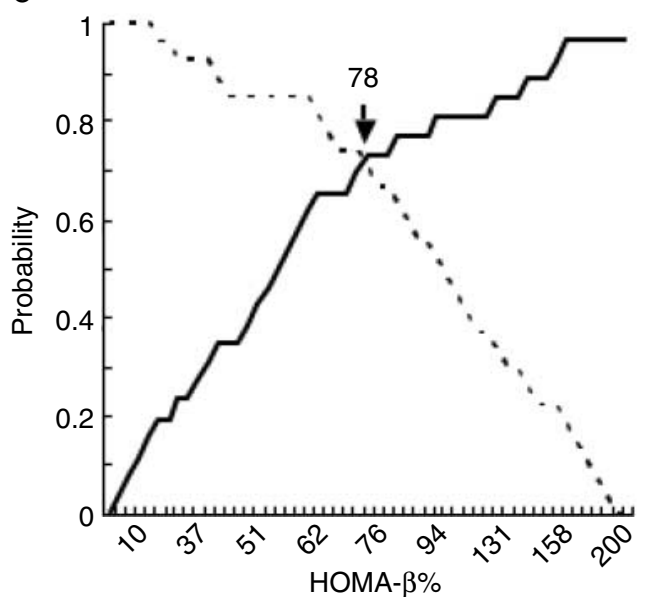

Figure 3 Association of $\beta$-cell function with improved glucose tolerance after surgery. (A) ROC curve analysis of IGI (solid line) and HOMA-\% $\beta$ (dotted line). (B) A cut-off value of 0.50 for IGI corresponded to a specificity (solid line) and sensitivity (dotted line) of $75.3 \%$. To convert values of IGI to $\mathrm{pM} / \mathrm{mM}$, multiply by 125 . (C) A cut-off value of 78 for HOMA- $\% \beta$ corresponded to a sensitivity (dotted line) and specificity (solid line) of $71.6 \%$. associated with improved glucose metabolism; IGI was identified $(P<0.05)$, but HOMA-\% $\beta$ and HOMA-IR were not. If one had 1 Unit higher IGI than another, odds ratio for the improvement of glucose metabolism was 9.9. Next, ROC analysis was carried out to determine the appropriate cut-off value of the preoperative parameters for predicting restoration of normal glucose metabolism after surgery; the AUC for IGI was 0.80 (95\% confidence interval (CI), 0.67-0.92) and AUC for HOMA- $\% \beta$ was 0.75 (95\% CI, 0.61-0.88; Fig. 3A). Although they did not differ significantly, IGI with a cut-off value of 0.50 appeared to be a better predictor with a sensitivity and specificity of $75.3 \%$ (Fig. 3B) compared with HOMA- $\% \beta$ with a cut-off value of 78 and a sensitivity and specificity of $71.6 \%$ (Fig. 3C). Therefore, we determined that IGI $>0.50$ was the best clinical parameter to predict recovery of normal glucose metabolism after successful surgery for acromegaly in patients presenting with IGT or DM before surgery. Glucose tolerance was normalized after cure with surgery in $95 \%$ of patients with IGI $>0.80$, whereas $95 \%$ of patients with IGI $<0.21$ failed to recover normal glucose metabolism. When patients who received oral hypoglycemic agents were excluded from the analysis, 0.54 was the optimal cut-off value of IGI to predict restoration of normal glucose metabolism after surgery (sensitivity and specificity of $74.0 \%$ ), and 81 was the optimal cut-off value of HOMA- $\% \beta$ (sensitivity and specificity of $69.5 \%$ ).

\section{Discussion}

It is well established that acromegaly, caused by excess GH in adult patients, induces IR; thus, insufficient $\beta$-cell function could lead to the development of impaired glucose metabolism in acromegalic patients as in obese individuals, who typically exhibit IR. In the present study of 92 patients with acromegaly, patients with DM exhibited defects in $\beta$-cell function, as assessed by HOMA- $\% \beta$ and IGI, compared with patients with NGT or IGT. However, IR in DM patients, as assessed by HOMA-IR and HOMA-\%S, was similar to that in patients with NGT or IGT. Because a reciprocal relationship exists between HOMA-\%S and HOMA-IR, we used HOMA-\%S as an indicator of IR in this study. Parameters of IR and $\beta$-cell function did not differ between IGT and NGT patients, except that IGT was associated with lower IGI compared with NGT. IGI indicates rapid response of $\beta$-cells to glucose load, whereas HOMA- $\% \beta$ represents basal $\beta$-cell function. Thus, exacerbation of IR in acromegaly would predispose to DM when basal $\beta$-cell function is impaired, and predispose to IGT when basal $\beta$-cell function is preserved but rapid response of $\beta$-cells is disturbed. This finding is consistent with a previous study that demonstrated impaired $\beta$-cell function only in acromegalic patients with abnormal glucose tolerance but not in those with NGT (7). 
Unexpectedly, serum levels of GH and IGF1 did not correlate with parameters of IR, other than a weak correlation between IGF1 and HOMA-\%S. Since both HOMA-IR and HOMA-\%S were significantly improved in all groups after pituitary surgery, the GH/IGF1 axis was surely involved in the exacerbation of IR, but their correlation may not be linear.

We next explored effects of the surgical cure of acromegaly on glucose metabolism in the same patients. We found that NGT was restored in $27 / 53(51 \%)$ of patients with IGT or DM immediately after cure of acromegaly. Parameters of IR were improved after surgery in all groups, but indicators of $\beta$-cell function were decreased in groups I and II but not in group III. Postoperative parameters of IR were similar among the patient groups, but indicators of $\beta$-cell function remained lower in group III than in the other patient groups. These observations suggest that improving IR, but not $\beta$-cell function, contributes to the restoration of normal glucose metabolism, and that patients with insufficient $\beta$-cell function before surgery do not achieve NGT even upon cure of acromegaly.

Lastly, we attempted to identify clinical parameters that could predict whether impaired glucose metabolism in an acromegalic patient was reversible. We found that some preoperative parameters (indicators of $\beta$-cell function and IR) differed between groups II and III. Logistic regression analysis demonstrated that only IGI was significantly associated with improved glucose metabolism in patients with IGT or DM before surgery. ROC analysis indicated that IGI of 0.50 before surgery was the best cut-off value to predict the patients in whom glucose metabolism will be restored to normal from IGT or DM. However, this cut-off value of IGI may depend on BMI and ethnicity. Patients examined in the present study tended to be lean than obese, and none had a BMI higher than 30. In addition, $\beta$-cell function in Asian populations, including Japanese people, is known to be lower than that observed among Caucasians. Thus, 0.50 as the cut-off value of IGI to predict normalization of glucose metabolism after surgery for acromegaly may be appropriate only for lean patients and/or Japanese patients.

Among the Japanese patients with acromegaly evaluated in the present study, BMI correlated positively with HOMA- $\% \beta$ and HOMA-IR, but was not associated with restoration of normal glucose metabolism after surgery. However, it is important to remember that there were no obese patients in the present study. Inclusion of obese patients may have resulted in BMI influencing whether glucose metabolism was normalized after cure with surgery, and the cut-off value of IGI would have to be adjusted with BMI.

Although IR is common in acromegaly, few studies report the effect of $\mathrm{GH}$ and IGF1 on IR, and their results were are not consistent (7-10). Kasayama et al. (7) and Fukuoka et al. (8) reported no correlation between IR and serum GH or IGF1 levels. In contrast,
Stelmachowska-Banas et al. (9) and Puder et al. (10) reported their associations. In our study, one indicator of IR, HOMA-IR, was not correlated with GH or IGF1 in a linear regression analysis, and the other indicator, HOMA-\%S, was only weakly correlated with IGF1. Together with previous studies, our results suggest that the GH/IGF1 axis and IR are not closely correlated in Japanese patients with acromegaly, even if acromegaly is involved in the increase in clinical parameters of IR.

A few studies have addressed the issue of $\beta$-cell function in acromegaly. Among them, Kasayama et al. (7) failed to demonstrate a correlation between $\beta$-cell function and GH or IGF1 levels, whereas Fukuoka et al. (8) detected a weak correlation between IGI and IGF1 levels. Long-standing IR appears to cause $\beta$-cell 'exhaustion', which leads to impaired $\beta$-cell function and the development of type $2 \mathrm{DM}$ in non-acromegalic subjects $(17,18)$. Although our results suggest that the GH/IGF1 axis is not directly involved in the impairment of $\beta$-cell function, we should consider this possibility, because acromegaly is typically not diagnosed for more than 10 years. Unfortunately, however, we could not precisely evaluate the duration of acromegaly in the present study to explore this issue.

In conclusion, we found that IR in non-obese Japanese patients with acromegaly was involved in the impairment of glucose metabolism. Both of IR and disturbed glucose metabolism were restored immediately after cure of acromegaly, if $\beta$-cell function was not deteriorated. Once $\beta$-cell function was impaired, abnormal glucose metabolism failed to normalize even after cure of acromegaly. For non-obese Japanese patients with acromegaly, a preoperative value of IGI $>0.50$ appears to be the best predictor of restoration of normal glucose metabolism upon surgical cure of the disease and the best indicator for preservation of $\beta$-cell function.

\section{Declaration of interest}

The authors declare that there is no conflict of interest that could be perceived as prejudicing the impartiality of the research reported.

\section{Funding}

This work was supported in part by grants from Okinaka Memorial Institute for Medical Research and Ministry of Health, Labor, and Welfare of Japan.

\section{References}

1 Ben-Shlomo A \& Melmed S. Acromegaly. Endocrinology and Metabolism Clinics of North America 200837 101-122. (doi:10. 1016/j.ecl.2007.10.002)

2 Holdaway IM. Excess morality in acromegaly. Hormone Research 200768 (Supplement 5) 166-172. (doi:10.1159/000110617)

3 Colao A, Ferone D, Marzullo P \& Lombardi G. Systemic complications of acromegaly: epidemiology, pathogenesis, and management. Endocrine Reviews 200425 102-152. (doi:10.1210/er.2002-0022) 
4 Resmini E, Minuto F, Colao A \& Ferone D. Secondary diabetes associated with principal endocrinopathies: the impact of new treatment modalities. Acta Diabetologica 200946 85-95. (doi:10. 1007/s00592-009-0112-9)

5 Kreze A, Kreze-Spirova E \& Mikulecky M. Risk factors for glucose intolerance in active acromegaly. Brazilian Journal of Medical and Biological Research 200134 1429-1433. (doi:10.1590/S0100879X2001001100009)

6 Nabarro JD. Acromegaly. Clinical Endocrinology $1987 \quad 26$ 481-512. (doi:10.1111/j.1365-2265.1987.tb00805.x)

7 Kasayama S, Otsuki M, Takagi M, Saito H, Sumitani S, Kouhara H, Koga M, Saitoh Y, Ohnishi T \& Arita N. Impaired $\beta$-cell function in the presence of reduced insulin sensitivity determines glucose tolerance status in acromegalic patients. Clinical Endocrinology 200052 549-555. (doi:10.1046/j.1365-2265.2000.00986.x)

8 Fukuoka H, Takahashi Y, Iida K, Kudo T, Nishizawa H, Imanaka M, Takeno R, Iguchi G, Takahashi K, Okimura Y, Kaji H \& Chihara K. Low serum IGF-1/GH ratio is associated with abnormal glucose tolerance in acromegaly. Hormone Research 200869 165-171. (doi:10.1159/000112590)

9 Stelmachowska-Banas M, Zdunowski P \& Zglicaynski W. Abnormalities in glucose homeostasis in acromegaly. Does the prevalence of glucose intolerance depend on the level of activity of the disease and the duration of the symptoms? Endokrynologia Polska 200960 20-24.

10 Puder JJ, Nilavar S, Post KD \& Freda PU. Relationship between disease-related morbidity and biochemical markers of activity in patients with acromegaly. Journal of Clinical Endocrinology and Metabolism 2005 90 1972-1978. (doi:10.1210/jc.2004-2009)

11 Giustina A, Barkan A, Casanueva FF, Cavagnini F, Frohman L, Ho K, Veldhuis J, Wass J, Von Werder K \& Melmed S. Criteria for cure of acromegaly: a consensus statement. Journal of Clinical Endocrinology and Metabolism 200085 526-529. (doi:10.1210/ jc. 85.2.526)
12 Levy JC, Matthews DR \& Hermans MP. Correct homeostasis model assessment (HOMA) evaluation uses the computer program. Diabetes Care 199821 2191-2192. (doi:10.2337/diacare.21.12. 2191)

13 Wallace TM, Levy JC \& Matthews DR. Use and abuse of HOMA modeling. Diabetes Care 200427 1487-1495. (doi:10.2337/ diacare.27.6.1487)

14 Seltzer HS, Allen EW, Herron AL Jr \& Brennan MT. Insulin secretion in response to glycemic stimulus: relation of delayed initial release to carbohydrate intolerance in mild diabetes mellitus. Journal of Clinical Investigation $1967 \mathbf{4 6}$ 323-335. (doi:10.1172/JCI105534)

15 Hanley JA \& McNeil BJ. The meaning and use of the area under a receiver operating characteristic (ROC) curve. Radiology 1982 $14329-36$.

16 Hanley JA \& McNeil BJ. A method of comparing the areas under receiver operating characteristic curves derived from the same cases. Radiology 1983148 839-843.

17 De Fronzo RA, Bonadonna RC \& Ferrannini E. Pathogenesis of NIDDM. A balanced overview. Diabetes Care 199215 318-368. (doi:10.2337/diacare.15.3.318)

18 Funakoshi S, Fujimoto S, Hamasaki A, Fujiwara H, Fujita Y, Ikeda K, Hamamoto Y, Hosokawa M, Seino Y \& Inagaki N. Analysis of factors influencing pancreatic $\beta$-cell function in Japanese patients with type 2 diabetes: association with body mass index and duration of diabetic exposure. Diabetes Research and Clinical Practice 200882 353-358. (doi:10.1016/j.diabres. 2008.09.010)

Received 15 January 2011

Accepted 1 February 2011 